\title{
Octants Are Cover-Decomposable
}

\author{
Balázs Keszegh • Dömötör Pálvölgyi
}

Received: 4 May 2011 / Revised: 8 September 2011 / Accepted: 27 September 2011 /

Published online: 19 October 2011

(C) Springer Science+Business Media, LLC 2011

\begin{abstract}
We prove that octants are cover-decomposable; i.e., any 12-fold covering of any subset of the space with a finite number of translates of a given octant can be decomposed into two coverings. As a corollary, we obtain that any 12-fold covering of any subset of the plane with a finite number of homothetic copies of a given triangle can be decomposed into two coverings. We also show that any 12 -fold covering of the whole plane with the translates of a given open triangle can be decomposed into two coverings. However, we exhibit an indecomposable 3-fold covering with translates of a given triangle.
\end{abstract}

Keywords Cover-decomposability · Geometric hypergraph coloring

\section{Introduction}

Let $\mathcal{P}=\left\{P_{i} \mid i \in I\right\}$ be a collection of geometric sets in $\mathbb{R}^{d}$. We say that $\mathcal{P}$ is an $m$ fold covering of a set $S$ if every point of $S$ is contained in at least $m$ members of $\mathcal{P}$. A 1 -fold covering is simply called a covering.

Definition A geometric set $P \subset \mathbb{R}^{d}$ is said to be cover-decomposable if there exists a (minimal) constant $m=m(P)$ such that every $m$-fold covering of any subset of $\mathbb{R}^{d}$ with a finite number of translates of $P$ can be decomposed into two coverings of the same subset. Define $m$ as the cover-decomposability constant of $P$. We note that in the literature the definition is slightly different and the notion defined here is

B. Keszegh ( $\square)$

Alfréd Rényi Institute of Mathematics, Budapest, Hungary

e-mail: keszegh@renyi.hu

D. Pálvölgyi

Eötvös University, Budapest, Hungary 
sometimes called finite-cover-decomposable; however, to avoid unnecessary complications, we simply call it cover-decomposable.

The simplest objects to examine are the orthants of $\mathbb{R}^{d}$. It is easy to see that a quadrant (two-dimensional orthant) is cover-decomposable. Cardinal [4] noticed that orthants in four and higher dimensions are not cover-decomposable as there is a plane on which their trace can be any family of axis-parallel rectangles, and it was shown by Pach, Tardos, and Tóth [12] that such families might not be decomposable into two coverings. Cardinal asked whether octants (three-dimensional orthants) are coverdecomposable. Our main result is an affirmative answer (the proof is given in Sect. 2).

Theorem 1 Octants are cover-decomposable; i.e., any 12-fold covering of any subset of $\mathbb{R}^{3}$ with a finite number of translates of a given octant can be decomposed into two coverings.

The intersection of the translates of the octant containing $(-\infty,-\infty,-\infty)$ with the $x+y+z=0$ plane gives the homothetic copies of an equilateral triangle. Since any triangle can be obtained by an affine transformation of the equilateral triangle we obtain the following.

Corollary 2 Any 12-fold covering of any subset of the plane with a finite number of homothetic copies of any given triangle can be decomposed into two coverings.

We say that a covering is locally finite if every compact set intersects only a finite number of covering sets, i.e., homothetic copies of the given triangle, in our case. Using standard compactness arguments, the previous corollary implies the following (the proofs are given in Sect. 4).

Theorem 3 Any locally finite 12-fold covering of the whole plane with homothetic copies of a triangle is decomposable into two coverings.

Theorem 4 Any 23-fold covering of the whole plane with homothetic copies of an open triangle is decomposable into two coverings.

The analogs of Corollary 2 and Theorem 3 for translates of a given triangle were proved with a bigger constant by Tardos and Tóth [17] using a more complicated argument (the original proof gave $m=43$, which was later improved by Ács [1] to $m=19$, which is still worse than our 12). Following their idea, using Theorem 3 for translates of a given open triangle, we obtain the following.

Corollary 5 Any 12-fold covering of the whole plane with the translates of an open triangle is decomposable into two coverings.

Our result brings the task to determine the exact cover-decomposability constant of triangles in range. Tardos and Tóth state that they cannot even rule out the possibility that the cover-decomposability constant of triangles is 3 . To complement our upper 
bound, in Sect. 3 we show a construction proving that the constant is actually at least 4.

Our proof of Theorem 1 in fact proves the following equivalent, dual form of Theorem 1.

Theorem 6 Any finite set of points in $\mathbb{R}^{3}$ can be colored with two colors such that any translate of a given octant with at least 12 points contains both colors.

To see that Theorem 1 implies Theorem 6, assume that the octant we fixed is the one containing $(-\infty,-\infty,-\infty)$ (the negative octant). Now for each vertex $v$, take the octant $V$ having apex $v$ and containing $(\infty, \infty, \infty)$ (a positive octant). Now a negative octant $O$ contains $v$ if and only if the positive octant $V$ contains $o$, the apex of $O$. As containment is preserved, coloring the positive octants with apices of the original point set according to Theorem 1, the same coloring for the vertices gives a valid coloring for Theorem 6 . The reverse implication is similar; it again uses that containment is preserved by this dualization (for more on dualization see the surveys [14] and [11]).

Finally, we mention the dual of Corollary 2, which is not equivalent to Corollary 2 but follows from Theorem 6 in the same way as Corollary 2 follows from Theorem 1.

Corollary 7 Any finite planar point set can be colored with two colors such that any homothetic copy of a given triangle that contains at least 12 points contains both colors.

We finally note that in this paper in many theorems it does not matter whether the respective underlying set (orthant, triangle, etc.) is open or not, and in the proofs, for simplicity, we consider the sets to be open (unless otherwise stated). Also, although the arrangement of the points is arbitrary, for simplicity, in the proofs we suppose that the objects are in general position, as a slight perturbation only increases the constraints that we have to satisfy.

For more about handling these issues and other results on cover-decomposability, see the recent surveys [14] and [11] and the papers [2, 3, 5, 9, 10, 13, 15-17].

\section{Proof of Theorems 1 and 6}

Denote by $W$ the octant with apex at the origin containing $(-\infty,-\infty,-\infty)$. We will work in the dual setting; that is, we have a finite set of points, $P$, in the space, that we want to color with two colors such that any translate of $W$ with at least 12 points contains both colors. We call such a two-coloring of a point set in the space a good coloring. If we can do such a coloring for any $P$, then it follows using a standard dualization argument (see [14] or [11]) that $W$ (and thus any octant) is cover-decomposable. So from now on our goal will be to show the existence of such a coloring.

For simplicity, suppose that no number occurs multiple times among the coordinates of the points of $P$ (otherwise, by a small perturbation of $P$ we can get such a 
point set, and its coloring will also be good for $P$ ). Denote the point of $P$ with the $t^{\text {th }}$ smallest $z$ coordinate by $p_{t}$ and the union of $p_{1}, \ldots, p_{t}$ by $P_{t}$. First we will show how to reduce the coloring of $P$ to a planar and thus more tractable problem.

Denote the projection of $P$ on the $z=0$ plane by $P^{\prime}$. Similarly denote the projection of $p_{t}$ by $p_{t}^{\prime}$, the projection of $P_{t}$ by $P_{t}^{\prime}$, and the projection of $W$ by $W^{\prime}$. Therefore, $W^{\prime}$ is the quadrant with apex at the origin containing $(-\infty,-\infty)$.

For such an ordered planar point set $P^{\prime}$ we say that a coloring with two colors is a good coloring, if for any $t$ and any translate of $W^{\prime}$ containing at least 12 points of $P_{t}^{\prime}$, it is true that the intersection of this translate and $P_{t}^{\prime}$ contains both colors. To see why we use the same notation for two differently defined colorings, the next claim shows that good coloring of a spatial point set and good coloring of the corresponding planar point set are equivalent problems.

Claim 8 The planar point set $P^{\prime}$ has a good coloring if and only if the spatial point set $P$ has a good coloring.

Proof Clearly, if we take a translate of $W$ with apex $w$ having $z$ coordinate bigger than the $z$ coordinate of $p_{t}$ and smaller than the $z$ coordinate of $p_{t+1}$, then the projection of the intersection of this translate with $P$ is equal to the intersection of $P_{t}^{\prime}$ with the translate of $W^{\prime}$ having apex $w^{\prime}$, the projection of $w$. Thus, having a good coloring for one problem gives a good coloring for the other if we give $p_{t}$ and $p_{t}^{\prime}$ the same color for every $t$.

Now we will prove that any $P^{\prime}$ has a good coloring, thus establishing Theorem 6 and, since they are equivalent, also Theorem 1 . To avoid going mad, we will omit the apostrophe in the following, so we will simply write $W$ instead of $W^{\prime}$ and so on. Also, we will use the term wedge to denote a translate of $W$.

A possible way to imagine this planar problem is that in every step $t$ we have a set of points, $P_{t}$, and our goal is to color the coming new point, $p_{t+1}$, such that we always have a good coloring. We note that this would be impossible in an online setting, i.e., without knowing in advance which points will come in which order. But using the fact that we know in advance every $p_{i}$ makes the problem solvable.

We start by introducing some notation. If $p_{x}<q_{x}$ but $p_{y}>q_{y}$ then we say that $p$ is NW from $q$ and $q$ is SE from $p$. In this case we call $p$ and $q$ incomparable. Similarly, $p$ is SW from $q$ (and $q$ is NE from $p$ ) if and only if both coordinates of $p$ are smaller than the respective coordinates of $q$.

Instead of coloring the points, we will rather define on them a bipartite graph $G$, whose proper two-coloring will give us a good coloring. Actually, as we will later see, this graph will be a forest.

We define $G$ recursively, starting with the empty set and the empty graph. At any step $j$ we define a graph $G_{j}$ on the points of $P_{j}$ and also maintain a set $S_{j}$ of pairwise incomparable points, called the staircase. Thus, before the $t^{\text {th }}$ step we have a graph $G_{t-1}$ on the points of $P_{t-1}$ and a set $S_{t-1}$ of pairwise incomparable points. In the $t^{\text {th }}$ step we add $p_{t}$ to our point set obtaining $P_{t}$, and we will define the new staircase, $S_{t}$, and also the new graph, $G_{t}$, which will have $G_{t-1}$ as a subgraph. Before the exact definition of $S_{t}$ and $G_{t}$, we make some more definitions and fix some properties that will be maintained during the process. 
In any step $j$, we say that a point $p$ is good if any wedge containing $p$ already contains two points of $P_{j}$ connected by an edge of $G_{j}$; i.e., at any time after $j$ a wedge containing $p$ will contain points of both colors in the final coloring.

At any time $j$, consider the order of the points of $S_{j}$ given by their $x$ coordinates. If two points of $S_{j}$ are consecutive in this order, then we say that these staircase points are neighbors. We note that this does not mean that they are connected in the graph. A point $s$ of the staircase is almost good if for at least one of its neighbors $s^{\prime}$ it is true that any wedge containing $s$ and $s^{\prime}$ contains two points of $P_{j}$ connected by an edge of $G_{j}$. Notice that the good points and the neighbors of the good points are always almost good.

We say that a point $p$ of $P_{j}$ is above the staircase if there exists a staircase point $s \in S_{j}$ such that $p$ is NE from $s$. If $p$ is not above or on the staircase, then we say that $p$ is below the staircase. Now we can state the properties we maintain.

At any time $j$ :

1. All points above the staircase are good.

2. All points of the staircase except the first and last are almost good.

3. All points below the staircase are incomparable.

4. If a wedge only contains points that are below the staircase, then it contains at most 3 points.

For $t=0$ all these properties are trivially true. Suppose that the properties hold at time $t-1$. Now we proceed with point $p_{t}$ according to the following algorithm maintaining all the properties. During the process, we denote the current graph by $G$ and the current staircase by $S$.

\section{Algorithm Step $t$}

Set $G=G_{t-1}$ and $S=S_{t-1}$.

Step (a) If $p_{t}$ is above the staircase $S_{t-1}$, then we do the following; otherwise skip to Step (b).

In this case $S_{t}=S_{t-1}$ and $G_{t}=G_{t-1} \cup\{e\}$, where $e$ is an arbitrary edge between $p_{t}$ and a point $s$ of $S_{t-1}$ which is $\mathrm{SW}$ from $p_{t}$. The properties will hold trivially by induction; the only thing we need to check is if $p_{t}$ is a good point, but this is again guaranteed as any wedge containing $p_{t}$ contains the edge $e$. The algorithm terminates.

Note that we proceed further if and only if $p_{t}$ is below the staircase $S_{t-1}$.

Step (b) If there exist two points $p$ and $q$ that are below the staircase and $p$ and $q$ are comparable, then we do the following; otherwise skip to Step (c).

Without loss of generality suppose that $q$ is SW from $p$. Notice that because of Property 3, either $p$ or $q$ is the last added point and there are no points below the staircase that are NE from $p$. Now, define the new staircase, $S$, as $S$ minus the points of $S$ that are NE from $p$, plus the point $p$. This way the points of the staircase remain pairwise incomparable, as we added $p$ and deleted all the points that were comparable to $p$. Also, we add the edge $p q$ to the graph, i.e. $G:=G \cup\{p q\}$. For an illustration of repeated application of this step, see Fig. 1 (edges of $G$ are drawn red). Thus, any wedge containing $p$ contains the edge $p q$, i.e. $p$ is a good point. Property 1 


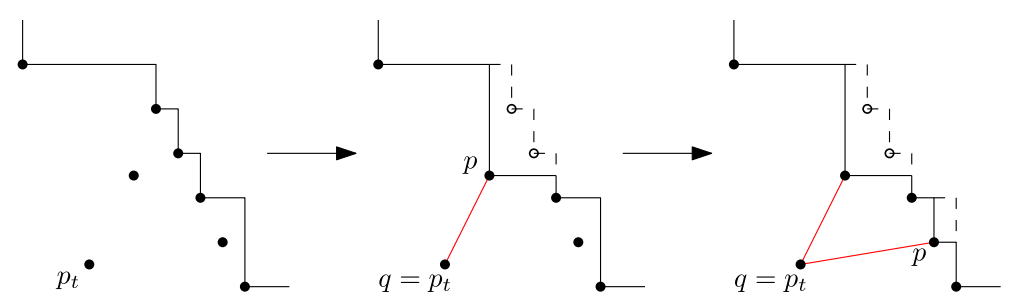

Fig. 1 Repeated application of Step (b) of the algorithm

is true for the points that were above the old $S$ by induction. All other points above $S$ are exactly the points that were deleted from the staircase in this step. All such points are NE from $p$ and thus any wedge containing them contains the edge $p q$. Property 2 holds for $p$ as it is a good point, and it holds for the 2 neighbors of $p$ as any point neighboring a good point is an almost good point. For any other $s$ from the staircase its neighbors remain the same, so it remains almost good.

Go back to Step (b) until Property 3 is satisfied, then proceed to Step (c).

Step (c) If there exist 4 points below the staircase such that these 4 points are pairwise incomparable and there exists a wedge $V$ such that $V$ contains these 4 points but no points of the staircase, then do the following; otherwise skip to Step (d).

Denote these 4 points by $q_{1}, q_{2}, q_{3}, q_{4}$ in increasing order of their $x$ coordinates. Notice that there are no points below the staircase that are comparable because of Step (b). Now define the new $S$ as the old $S$ minus the points of $S$ that are NE from $q_{2}$ or $q_{3}$, plus the points $q_{2}$ and $q_{3}$. This way the points of the staircase remain pairwise incomparable as we added $q_{2}$ and $q_{3}$ and deleted all the points that were comparable to them. Also, we add the edges $q_{1} q_{2}$ and $q_{3} q_{4}$ to the graph, i.e. $G_{t}=G_{t-1} \cup\left\{q_{1} q_{2}, q_{3} q_{4}\right\}$. For an illustration see Fig. 2(a). Property 1 is true for the points that were above the old $S$ by induction. All other points above the new $S$ are exactly the points that were deleted from the staircase in this step. It is easy to check if such a point is either NE from both of $q_{1}$ and $q_{2}$ or it is NE from both of $q_{3}$ and $q_{4}$ (we use that $V$ was completely below the staircase, see Fig. 2(b)). Thus, a wedge containing such a point contains the edge $q_{1} q_{2}$ or the edge $q_{3} q_{4}$, and Property 1 will be true. If $q_{2}$ has a preceding neighbor, then Property 2 is true for $q_{2}$ and also for its neighbor which is not $q_{3}$ as a wedge covering them must cover $q_{1}$ as well and thus the edge $q_{1} q_{2}$, i.e. they are almost good. If $q_{2}$ becomes the first point of the staircase, then we don't need Property 2 to hold for $q_{2}$. By symmetry $q_{3}$ is either the last point of the staircase or $q_{3}$ and its neighbor which is not $q_{2}$ are also almost good. For any other $s$ from the rest of the staircase (except the first and last point), $s$ remains almost good by induction as its neighbors do not change.

Go back to Step (c) until Property 4 is satisfied, then proceed to Step (d).

Step (d) Set $S_{t}=S$ and $G_{t}=G$ and the algorithm terminates. 


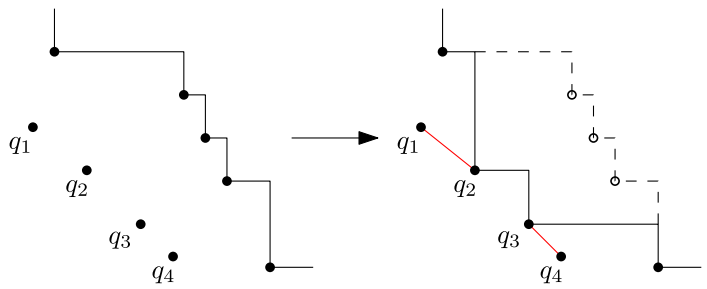

(a)

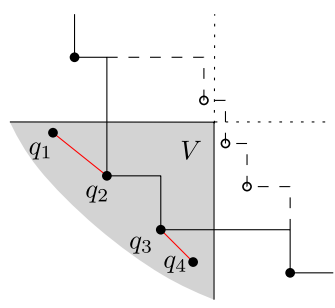

(b)

Fig. 2 Application of Step (c) of the algorithm

Fig. 3 At most 11 points can be in a monochromatic wedge

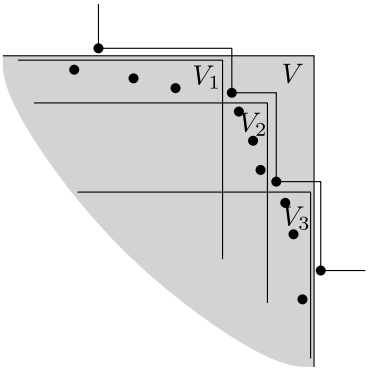

Adding $p_{t}$ below the staircase and proceeding as in Step (b) or Step (c) always maintains Properties 1 and 2. As neither Step (b) nor Step (c) can be applied anymore, Properties 3 and 4 must hold as well. Now let us examine the graph $G$.

Claim 9 The final graph $G$ is a forest.

Proof We prove by induction a stronger statement, that $G$ will be such a forest that the components of the points below the staircase are disjoint trees.

When we add an edge in Step (a), then the newly added point that goes above the staircase will be one of the endpoints; thus this property is maintained.

When we add an edge in Step (b) or (c), then it connects two points below the staircase, one of which we immediately move to the staircase, so we are done by induction.

Claim 10 Any two-coloring of $G$ is a good coloring of $P$.

Proof Take an arbitrary two-coloring of $G$. Take an arbitrary wedge $V$ at time $t$ that contains at least 12 points of $P_{t}$. If $V$ contains a point from above the staircase $S_{t}$, then by Property $1 V$ contains points of both colors. If $V$ contains at least 3 points from the staircase, then $V$ also contains 3 consecutive points; thus by applying Property 2 to the middle one $V$ contains both colors (as it contains both neighbors of this middle point). Finally, if a wedge $V$ does not contain a point from above the staircase and contains at most 2 points from the staircase, then all the points below the staircase that 
are covered by $V$ can be covered by 3 wedges containing points only from below the staircase (see wedges $V_{1}, V_{2}$, and $V_{3}$ in Fig. 3). By Property 4 each of these wedges cover at most 3 points; thus $V$ can contain altogether at most 11 points ( 2 from the staircase and $3 \cdot 3$ from below the staircase), a contradiction.

The preceding claim finishes the proof of Theorem 6 and thus also of Theorem 1 .

As noted by our anonymous reviewer, when applying this algorithm in the special case of homothetic triangles in the plane, as the spatial point set is on the $x+y+z=0$ plane, Step (a) can never occur during the algorithm. Also, as in this special case no new point comes NE to a previous point, it is true for all edges of $G$ that its endvertices can be contained in an octant that contains no other points. Thus, when in the $x+y+z=0$ plane, for all edges of $G$ its end-vertices can be contained in a homothet of the fixed triangle that contains no other points. In other words, the final forest is a subgraph of the Delaunay-graph w.r.t. that fixed triangle (for direct applications of different Delaunay-graphs for such problems, see, e.g., [7]).

\section{Miscellany and a Lower Bound}

We have seen in the Introduction that if the point set of Theorem 6 is from the $x+y+z=0$ plane, then the problem is equivalent to the cover-decomposability of homothetic copies of an equilateral triangle. Another special case is if the point set is on the $x+y=0$ plane. The intersection family of the octants with this plane is the family of bottomless axis-parallel rectangles (a set is a bottomless axis-parallel rectangle if it is the homothetic copy of the set $\{(x, y): 0<x<1, y<0\})$. Bottomless rectangles were examined by the first author in [6], where it was proved that any 3 -fold covering with bottomless rectangles is decomposable into two coverings and also that any finite point set can be colored with two colors such that every bottomless rectangle containing at least 4 points contains both colors. It was also shown that these results are sharp. We will use the ideas from [6] to prove the following claims, the first of which is a strengthening of Theorem 6 in a special case and the second giving a sharp lower bound for this special case, which also holds for the general case.

Claim 11 If the projection of the original point set from $R^{3}$ onto the $z=0$ plane yields a point set $P$ having only pairwise incomparable points, then it admits a twocoloring such that any translate of a given octant that contains at least 4 points contains points with both colors.

Proof We use the same notation as in Sect. 2. Now at any time the points of $P_{t}$ are pairwise incomparable. Order them according to their $x$ coordinate. We will maintain a partial coloring such that, at any time $t$ :

1. There are no two consecutive points in this order that are not colored.

2. The colored points are colored alternatingly. 


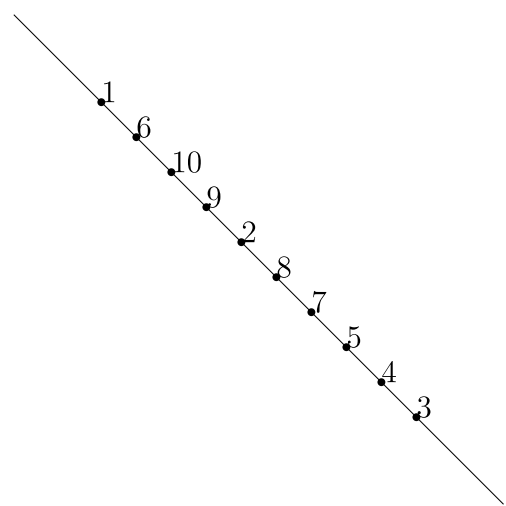

(a)

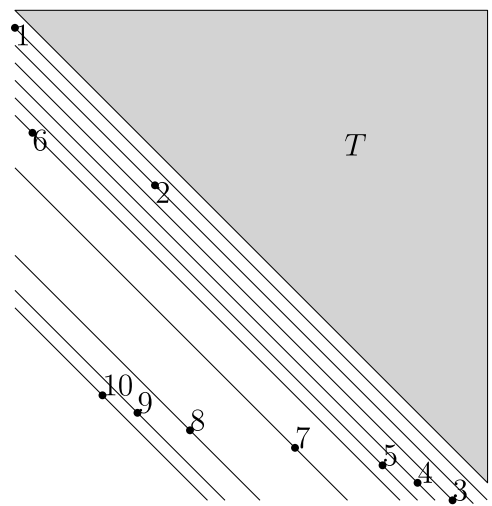

(b)

Fig. 4 Lower bound constructions

We start with the empty set and then in a general step we add the point $p_{t}$. If in the order it goes between two colored points, then we leave it uncolored. If it comes next to an uncolored point, then we color these two points maintaining the alternating coloring. At the end we color the remaining uncolored points arbitrarily. We claim that at any time $t$ any wedge covering at least 4 points covers points from both colors already at step $t$ of the coloring. Indeed, any wedge covers consecutive points; it covers at least 2 (consecutive) colored points by Property 1, and any two consecutive colored points are colored differently by Property 2 .

Claim 12 For any octant there exists a 10 point set $P \subset R^{3}$ such that its projection onto the $z=0$ plane yields a point set having only pairwise incomparable points, yet in any two-coloring of $P$ there exists a translate of a given octant that contains 3 points with the same color and no other points.

Proof The point set on Fig. 4(a) has the needed properties (for simplicity, the projection of the point having the $t^{\text {th }}$ biggest $z$ coordinate is denoted by $t$ instead of $p_{t}$ ). Indeed, suppose on the contrary that there exists a two-coloring with no monochromatic wedge covering exactly 3 points. It is easy to check that the triples $(1,2,3)$, $(1,2,4),(1,2,5),(3,4,5),(6,2,5),(6,2,7),(6,2,8),(5,7,8),(6,1,2),(6,1,9)$, $(6,1,10),(2,9,10)$ can all be covered by some wedge at some time $t$. By the pigeonhole principle there are two points from $(1,2,6)$ that have the same colors. If, e.g., 1 and 2 are colored red, then by the first three triples 3, 4, and 5 all must be colored blue, but then the fourth triple is monochromatic, a contradiction. The analysis is similar if 2 and 6 have the same color. Finally, if 1 and 6 are, e.g., red and 2 is blue, then we obtain a contradiction from the last three triples, as 9 and 10 should be both blue because of the penultimate and antepenultimate triples, but then the ultimate triple is monochromatic. 
This construction can be modified a bit to imply the same result for translates of a given triangle.

Claim 13 There exists a 10 point set $P \subset R^{2}$ and a given triangle $T$ such that in any two-coloring of $P$ there exists a translate of $T$ that contains 3 points of the same color and no other points.

Proof The point set and the triangle on Fig. 4(b) have the needed properties; the proof of this is exactly the same as that of the previous claim.

Finally we note that this construction is a bit smaller than the one in [6], which had size 12 , so we obtain a smaller construction for that problem too by taking the same points as in Claim 11 projected onto the $y=0$ plane.

\section{Coverings of the Whole Plane}

In this section we prove Theorem 3, Theorem 4, and Corollary 5.

To prove Theorem 3, we need the following well-known lemma.

Lemma 14 (König's Infinity Lemma, [8]) Let $V_{0}, V_{1}, \ldots$ be an infinite sequence of disjoint nonempty finite sets, and let $G$ be a graph on their union. Assume that every vertex $v_{n}$ in a set $V_{n}$ with $n \geq 1$ has a neighbor $f\left(v_{n}\right)$ in $V_{n-1}$. Then $G$ contains an infinite path, $v_{0} v_{1} \ldots$ with $v_{n} \in V_{n}$ for all $n$.

Proof of Theorem 3 We need to prove that any locally finite 12-fold covering of the whole plane with homothetic copies of a triangle is decomposable into two coverings. Take $K_{1} \subset K_{2} \subset \cdots$ compact sets such that their union is the whole plane. Let each $v_{n} \in V_{n}$ be a possible coloring of those finitely many triangles that intersect $K_{n}$ such that every point of $K_{n}$ is covered by both colors. In this case $V_{n}$ is nonempty because of Corollary 2. The function $f$ is the natural restriction to the triangles that intersect $K_{n-1}$. The infinite path gives a partition to two coverings.

Proof of Theorem 4 We need to prove that any 23-fold covering of the whole plane with homothetic copies of a triangle is decomposable into two coverings. First take a compact set $K_{1}$. Select $\mathcal{T}_{1}$, a family of finitely many homothetic copies of the triangle that already give a 12 -fold covering of $K_{1}$. Denote by $K_{1}^{\prime}$ the (open) set that is 12 fold covered by $\mathcal{T}_{1}$ and by $K_{1}^{*}$ the union of the triangles from $\mathcal{T}_{1}$. So we have $K_{1} \subset$ $K_{1}^{\prime} \subset K_{1}^{*}$. Take any coloring of $\mathcal{T}_{1}$ such that every point of $K_{1}^{\prime}$ is covered by both colors (such a coloring exists because of Corollary 2).

Now select a $K_{2}$ compact set such that $K_{1}^{*} \subset K_{2}$. Select $\mathcal{T}_{2}$, a family of finitely many homothetic copies of the triangle that already give a 12 -fold covering of the compact set $K_{2} \backslash K_{1}^{\prime}$. Note that such a family exists because the points outside $K_{1}^{\prime}$ are covered by at most 11 members of $\mathcal{T}_{1}$. Define $K_{2}^{\prime}$ as the set that is 12 -fold covered by $\mathcal{T}_{1} \cup T_{2}$ and by $K_{2}^{*}$ the union of the triangles from $\mathcal{T}_{1} \cup \mathcal{T}_{2}$. Take any coloring of $\mathcal{T}_{2}$ such that every point of $K_{2}^{\prime}$ is covered by both colors (such a coloring exists because of Corollary 2). 
Similarly define $K_{3}, \ldots$ such that their union is the whole plane. Since the $\mathcal{T}_{i}$ families are disjoint, we get a good coloring.

Finally, for completeness we show how Theorem 3 implies Corollary 5, using the ideas from [17].

Proof of Corollary 5 We need to prove that any 12-fold covering of the whole plane with translates of a triangle is decomposable into two coverings. Take any such covering $\mathcal{T}$; the integer grid defines a decomposition of the plane to integer squares. From the compactness of each such closed square it easily follows that there is a finite subset of $\mathcal{T}$ that is a 12 -fold cover of this square. Take the union of such finite coverings for all squares of the grid. It is easy to see that this subset $\mathcal{T}^{\prime}$ of $\mathcal{T}$ is a 12 -fold covering of the whole plane. We claim that $\mathcal{T}^{\prime}$ is also locally finite. Indeed, for an arbitrary compact set $K$, the translates of a given triangle that intersect $K$ can intersect only finitely many squares of the grid, and thus finitely many sets from $\mathcal{T}^{\prime}$.

\section{Remarks}

Several important questions remain open. Our method could only provide a decomposition into two coverings. Is it possible to decompose any covering of the space with octants/plane with homothets of a triangle into more than two coverings if the original covering is thick enough? Only some weaker bounds are known for related problems [18].

We still do not know anything about (infinite) coverings of the plane/space with translates of closed polygons/octants. Is it possible to decompose such coverings?

For more related questions, see the recent surveys [14] and [11].

Acknowledgements We would like to thank Jean Cardinal for calling our attention to this problem. We would also like to thank Máté Vizer for discussions and ideas related to Sect. 4 and an anonymous reviewer for several useful remarks. B. Keszegh was supported by OTKA NK 78439. The European Union and the European Social Fund have provided financial support to the project under the grant agreement no. TÁMOP 4.2.1./B-09/1/KMR-2010-0003.

\section{References}

1. B. Ács, Síkfedések szétbonthatósága. Master Thesis (in Hungarian). http://www.cs.elte.hu/blobs/ diplomamunkak/mat/2010/acs_bernadett.pdf

2. Aloupis, G., Cardinal, J., Collette, S., Langerman, S., Orden, D., Ramos, P.: Decomposition of multiple coverings into more parts. In: SODA, pp. 302-310 (2009)

3. Buchsbaum, A.L., Efrat, A., Jain, S., Venkatasubramanian, S., Yi, K.: Restricted strip covering and the sensor cover problem. In: SODA, pp. 1056-1063 (2007)

4. Cardinal, J.: personal communication

5. Gibson, M., Varadarajan, K.: Decomposing coverings and the planar sensor cover problem. arXiv:0905.1093v1

6. Keszegh, B.: Weak conflict-free colorings of point sets and simple regions. In: The 19th Canadian Conference on Computational Geometry (CCCG07), Proceedings, pp. 97-100 (2007)

7. Cardinal, J., Korman, M.: Coloring planar homothets and three-dimensional hypergraphs. arXiv:1101.0565 
8. König, D.: Theorie der Endlichen und Unendlichen Graphen, Kombinatorische Topologie der Streckenkomplexe. Akademie Verlag, Leipzig

9. Pach, J.: Decomposition of multiple packing and covering. In: 2. Kolloq. Diskrete Geometrie, Salzburg, pp. 169-178. Math. Inst. Univ. Salzburg, Salzburg (1980)

10. Pach, J.: Covering the plane with convex polygons. Discrete Comput. Geom. 1, 73-81 (1986)

11. Pach, J., Pálvölgyi, D., Tóth, G.: Survey on the decomposition of multiple coverings. To appear

12. Pach, J., Tardos, G., Tóth, G.: Indecomposable coverings. Can. Math. Bull. 52, 451-463 (2009)

13. Pach, J., Tóth, G.: Decomposition of multiple coverings into many parts. In: 23rd ACM Symposium on Computational Geometry, pp. 133-137. ACM Press, New York (2007). Also in: Discrete Comput. Geom. 42, 127-133 (2009)

14. Pálvölgyi, D.: Decomposition of geometric set systems and graphs. Ph.D. thesis. arXiv:1009.4641

15. Pálvölgyi, D.: Indecomposable coverings with concave polygons. Discrete Comput. Geom. 44(3), 577-588 (2010)

16. Pálvölgyi, D., Tóth, G.: Convex polygons are cover-decomposable. Discrete Comput. Geom. 43(3), 483-496 (2010)

17. Tardos, G., Tóth, G.: Multiple coverings of the plane with triangles. Discrete Comput. Geom. 38, 443-450 (2007)

18. Varadarajan, K.: Weighted geometric set cover via quasi-uniform sampling. In: STOC, pp. 641-648 (2010) 